\title{
Determination of iodine value of palm olein mixtures using differential scanning calorimetry
}

\begin{abstract}
Differential scanning calorimetry (DSC) was used to determine the iodine value (IV) of various palm olein $(\mathrm{PoO})$ mixtures. Eight different $\mathrm{PoO}$ mixtures, namely, PoO:PKO, PoO:CoO, PoO:PS, PoO:PO, PoO:CaO, PoO:OeO, PoO:CnO and PoO:SFO were prepared at different ratios (w/w) to give various IV (PKO represents palm kernel olein; CoO, coconut oil; PS, palm stearin; $\mathrm{PO}$, palm oil; $\mathrm{CaO}$, canola oil; $\mathrm{OeO}$, olive oil; $\mathrm{CnO}$, corn oil; and SFO, sunflower oil). Each sample was then scanned from 80 to $-100{ }^{\circ} \mathrm{C}$ at $-5^{\circ} \mathrm{C} / \mathrm{min}$ using a DSC. All the mixtures showed two exothermic peaks in their cooling thermograms, except PoO:SFO mixtures which showed three peaks. Results of stepwise multiple linear regression (SMLR) analysis showed that five independent variables extracted from each of these peaks, namely, on-set temperature, off-set temperature, peak temperature, peak height and peak enthalpy could predict well the IV of each mixture. The calibration models developed showed appreciable effectiveness, re-producibility and accuracy, and specificity towards the calibration data set. A shared calibration model for each group of $\mathrm{PoO}$ mixtures i.e. highlauric (PoO:PKO and PoO:CoO), high-palmitic (PoO:PS and $\mathrm{PoO}: \mathrm{PO}$ ) and high-oleic (PoO:CaO and PoO:OeO) mixtures was also developed. SMLR analysis showed that the shared models were also capable in predicting IV of the PoO mixtures, even though the coefficient of determination, R2, was slightly lower than that of their individual models. The shared calibration models also had good reproducibility and accuracy when compared with the standard chemical method. In conclusion, DSC provides an effective method in determining IV for routine analysis in the industries, whereby one single model could be calibrated for the use of all oil and fat products that have similar chemical compositions such as high-lauric, high-palmitic or high-oleic mixtures in the industry.
\end{abstract}

Keyword: Calibration model; Differential scanning calorimetry; Iodine value; Palm olein mixtures 